\title{
Distributed Group-Based Mobility Management Scheme in Wireless Body Area Networks
}

\author{
Moneeb Gohar, ${ }^{1}$ Hind Ahmed M. Alrubaish, ${ }^{2}$ \\ Ruba Suliman M. Alowaid, ${ }^{3}$ and Jin-Ghoo Choi ${ }^{4}$ \\ ${ }^{1}$ Department of Computer Science, Bahria University, Islamabad, Pakistan \\ ${ }^{2}$ Department of Computer, Deanship of Preparatory Year, University of Dammam, \\ Dammam, Saudi Arabia \\ ${ }^{3}$ University of Dammam, Dammam, Saudi Arabia \\ ${ }^{4}$ Department of Information and Communication Engineering, Yeungnam University, \\ Gyeongsan, Republic of Korea
}

Correspondence should be addressed to Moneeb Gohar; moneebgohar@gmail.com and Jin-Ghoo Choi; jchoi@yu.ac.kr

Received 2 February 2017; Revised 19 April 2017; Accepted 26 April 2017; Published 23 May 2017

Academic Editor: Jaime Lloret

Copyright (C) 2017 Moneeb Gohar et al. This is an open access article distributed under the Creative Commons Attribution License, which permits unrestricted use, distribution, and reproduction in any medium, provided the original work is properly cited.

For group-based mobility management in 6LoWPAN-based wireless body area networks (WBAN), some schemes using the Proxy Mobile IPv6 (PMIP) have been proposed. However, the existing PMIP-based mobility schemes tend to induce large registration delay and handover delay. To overcome such limitations, we propose a new distributed group-based mobility management scheme, in which the Local Mobility Anchor (LMA) function is implemented by each Mobile Access Gateway (MAG) and the handover operation is performed between two neighboring MAGs without the help of LMA. Besides, each MAG maintains the information of the group of mobile sensors and aggregates the Authentication-Authorization-Accounting (AAA) query messages for a group of mobile sensors as a "single" message to decrease the control overhead. By numerical analysis, it is shown that the proposed scheme can reduce the registration and handover delays, compared to the existing PMIP-based mobility schemes.

\section{Introduction}

World population growth is facing challenges like increase of life expectancy leading to aging population and rise in healthcare costs. These challenges have triggered the introduction of novel technology-driven enhancements to current healthcare practices such as small and intelligent medical sensors which can be worn on or implanted in the human body. These sensors send their data to an external medical server. With these sensors, the patient experiences greater physical mobility. On the other hand, millions of people die from different fatal diseases like cancer and cardiovascular disease every year. This is because many people are diagnosed when it is too late. However, it is possible to utilize the latest technological advances in wireless body area network (WBAN) systems for the early detection and prevention of potential diseases that may occur later in people's lives. This can be done by using the sensors nodes on WBAN for continuous monitoring of health conditions [1-3].

In WBAN, sensors can be attached to the human body or clothes $[4,5]$. These sensors can be used to measure the parameters associated with the human body. The measured values can be gathered and transmitted to the main server healthcare applications.

The IP shortage problem can be solved through IPv6 addresses, because it has large address spaces. Recently, Low-power Wireless Personal Area Networks (LoWPANs) have attracted lots of attention because of the Internet of Things (IOTs). The Internet Engineering Task Force (IETF) has set up a working group recently for IPv6 over Lowpower Wireless Personal Area Networks (6LoWPANs) [6], in which it is possible to connect wireless sensor nodes to IPv6 networks. However, the wireless sensors nodes are unable to hold the complete IPv6 address because the maximum 
packet size of IEEE 802.15 .4 is 127 bytes. For this purpose, the 6LoWPAN adds an adaptation layer between MAC and the network layer to compress the 40-byte IPv6 packet header into 2 bytes for incoming communication and decompress it for the outgoing interface.

In mobile environments, it is required to provide sensor nodes with mobility management, such as handover control. The host-based approaches such as Mobile IPv6 (MIPv6) [7], Hierarchal Mobile IPv6 (HMIPv6) [8], and Fast Mobile IPv6 (FMIPv6) [9] are unsuitable to be applied in Internet Protocol (IP) based wireless sensor networks. All of the sensors have mobility stack and have actively participated in mobility related signaling. The network-based approaches, such as Proxy Mobile IPv6 (PMIPv6), can be considered as the most suitable approach for the mobility management in 6LoWPAN based WBAN.

Recently, several schemes have been proposed to support mobility in the 6LoWPAN-based WBAN [10, 11], which usually use the Proxy Mobile IPv6 (PMIP) protocol [12]. These schemes reduce the registration and handover delays, but there remains much room for improvement in the delay performance.

In this paper, we propose an enhanced group-based mobility scheme for 6LoWPAN-based WBAN by using the distributed PMIP approach so as to further reduce registration and handover delays. In the proposed scheme, the Local Mobility Anchor (LMA) function is implemented by each Mobile Access Gateway (MAG), and MAGs perform the handover control operations for the group of sensors. The MAGs also perform the aggregation of AuthenticationAuthorization-Accounting (AAA) query messages incurred by the movement of the group of sensor nodes. Different from the standard PMIP protocol, each MAG does not perform the binding update and deregistration operations with LMA, since it has the LMA functionality.

The rest of this paper is organized as follows. Section 2 describes the related works and existing mobility schemes for comparison and motivation. Section 3 describes the proposed distributed group-based mobility scheme. Section 4 demonstrates the performance analysis by comparing the proposed and existing schemes by numerical analysis. Section 5 presents the numerical results and discussion. Section 6 concludes this paper.

\section{Related Works}

In this section, first related works are described and then the existing group-based mobility schemes are considered for comparison in this paper.

2.1. Related Works. Reference [13] provides a survey of sensor devices and protocols on physical layer, data link layer, and radio technology aspects of body area networks. This paper also highlighted some of the design challenges and open issues on body area networks that still need to be addressed. In [14], it is shown that IPv6 over Low-power Wireless Personal Area Networks (6LoWPANs) is suitable for wireless body sensor networks because the sensors are based on IEEE 802.15.4 standard. The 6LoWPAN mobility has been an important solution in wireless body sensor networks. Most of the existing host-based mobility [7-9, 15] protocols for IPv6 are unsuitable for 6LoWPAN based wireless sensor networks, because they are tunnel based approaches, which shows that all of the mobile sensors actively participated in mobility related signaling in order to ensure the continuity of communications. The networkbased approach $[12,16]$ is suitable to handle the mobility management of 6LoWPAN based body sensors. On behalf of sensors, the network node exchanges the mobility related signaling and there is no need to perform Duplicate Address Detection (DAD) of the IP address and thus it reduces the control signaling cost and handover latency of each sensor. In [17], the author highlighted the requirements and resources for adapting the existing solutions to 6LoWPAN based on the notion that single mobile node's perspective is still a challenge and further researches are required. Therefore, [18] proposed a scheme to support Internet Protocol (IP) wireless sensor networks in which the packet format in ingress interface is to deal with the handoff procedure. In [17], it is also shown that multihop communications are not supported by Proxy Mobile IP (PMIP) protocol. Hence, in [19], a multihop communication scheme has been proposed in the ingress interface by using mesh routing. But this scheme is only on single mobile node perspective. So, if there is a group of mobile nodes moving around together, then this scheme [19] has no effective solution. In [20], a 6LoWPAN based mobility scheme has been proposed which depends on the dispatch types. But this scheme does not have a clear improvement on reducing handover delay. From the network-based perspective, in [21], a Network Mobility (NEMO) [10] based 6LoWPAN mobility scheme has been proposed, in which a mobile router performs the handoff and modifies the 6LoWPAN dispatch. However, this protocol overloaded the mobile router. To maintain the sessions while reducing the handover delay and signaling cost of group-based mobility in 6LoWPAN-based wireless sensor body area networks is an important issue. Therefore, [22] considered lots of correlated sensor nodes moving together and performed handoff together at the same time in PMIPv6 environment. The Local Mobility Anchor (LMA) calculates the SNR value for each sensor and makes a group of sensors with similar SNR values. This protocol sends Proxy Binding Ack (PBA) per group which can reduce the handoff signaling cost and save some additional unnecessary handoff messages such as Proxy Binding Update (PBU) and deregistration PBU (DeReg PBU). However, this protocol does not reduce the Router Solicitation (RS) and Router Advertisement (RA) messages in case body sensors move in the PMIPv6 domain together. Therefore, this protocol is not suitable for the 6LoWPAN-based wireless sensor body network. So, in [23], network architecture is proposed which integrates NEMO and 6LoWPAN. The mechanism of group mobility management and the signaling process containing the registration at the home network, association negotiation, handoff between different access routers, and packet routing are discussed. However, this protocol increases the burden of the sensor router due to connection and support for 


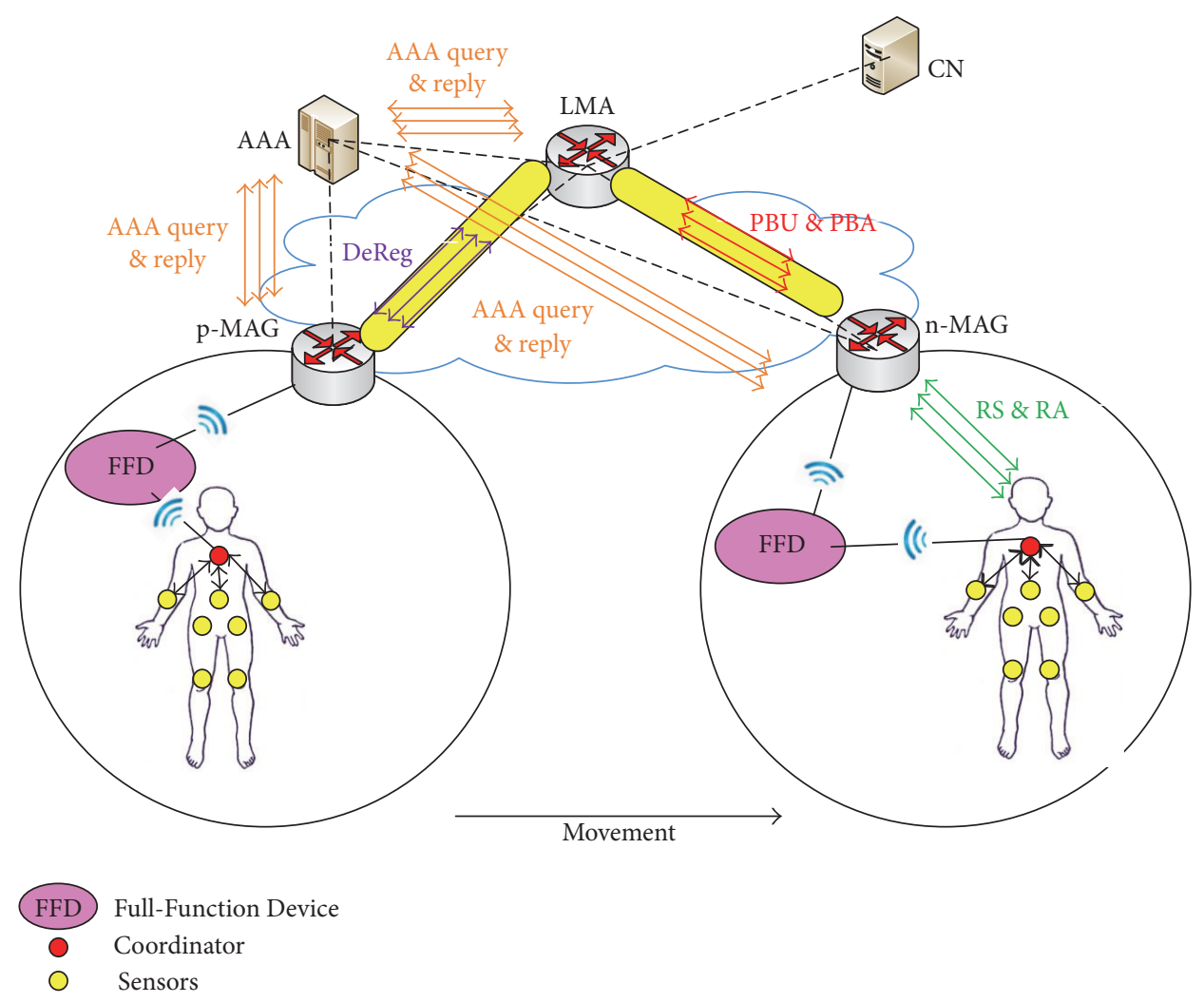

FIgURE 1: Existing PMIP mobility schemes.

other normal sensor nodes inside the group. This protocol is designed for MIPv6, not for PMIPv6.

\subsection{Existing Group-Based Mobility Schemes for Comparison}

2.2.1. PMIP [12]. In this scheme, the standard PMIP is used for the group-based mobility support [11]. As shown in Figure 1, when a set of body sensors are detached from a previous MAG ( $\mathrm{p}-\mathrm{MAG}$ ), the $\mathrm{p}-\mathrm{MAG}$ sends deregistration (DeReg) messages to LMA for each body sensor. When the body sensors are attached to a new MAG (n-MAG), each body sensor sends a Router Solicitation (RS) message to nMAG via the coordinator and Full-Function Device (FFD) which are preconfigured in the 6LoWPAN network [6].

Upon reception of RS messages from body sensors, nMAG will send the Authentication-Authorization-Accounting (AAA) query messages for authentication for all body sensors. After authentication, AAA server responds with AAA reply messages, containing the LMA address, to n-MAG. Then, n-MAG will send Proxy Binding Update (PBU) messages to LMA for each body sensor. Now, LMA will perform the AAA query operation with AAA server by exchanging $A A A$ query and reply messages for each sensor. After that, LMA sends Proxy Binding Ack (PBA) messages to n-MAG in response to each respective $P B U$ message. Finally, n-MAG responds with Router Advertisement (RA) messages to the body sensors.
2.2.2. PMIP-Group [10]. The standard PMIP-based scheme has a drawback that a lot of $D e R e g, P B U$, and $P B A$ messages are exchanged between LMA and MAGs (p-MAG and nMAG) for all body sensors.

To enhance this PMIP scheme [12], the PMIP-Group [10] was proposed, in which a single DeReg message is exchanged between p-MAG and LMA by aggregating the associated messages from all sensors, as shown in Figure 2. In case of handover to n-MAG, the $P B U$ and $P B A$ messages are also aggregated between LMA and n-MAG. By this aggregation, the number of $P B U$ and $P B A$ messages can be reduced between LMA and n-MAG.

2.2.3. PMIP-Coordinator [11]. The PMIP-Group [7] scheme tends to exchange many $R S / R A$ messages between sensors and MAGs (p-MAG and n-MAG). The PMIP-Coordinator scheme [11] was proposed for further enhancement of PMIPGroup scheme [10]. In this scheme, the coordinator will communicate with n-MAG on behalf of the body sensors, as shown in Figure 3.

When the coordinator is attached to n-MAG, then it sends a single Router Solicitation (RS) message, which contains the associated group information, MN-IDs, and link-layer address, to n-MAG, by way of FFD at a time. Then, n-MAG responds with a Router Advertisement (RA) message to the coordinator in response to the $R S$ message. 

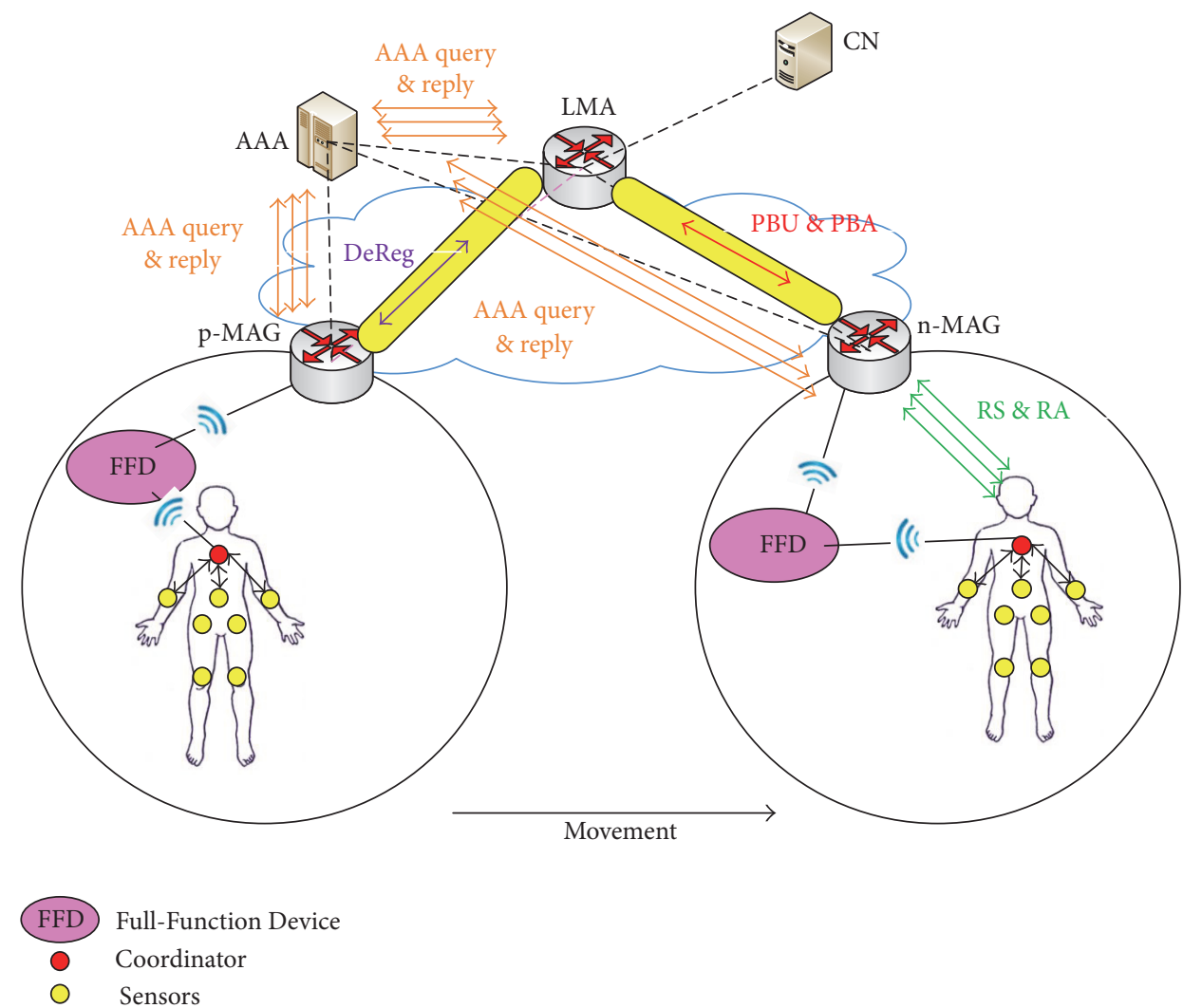

FIGURE 2: Existing PMIP-Group mobility schemes.

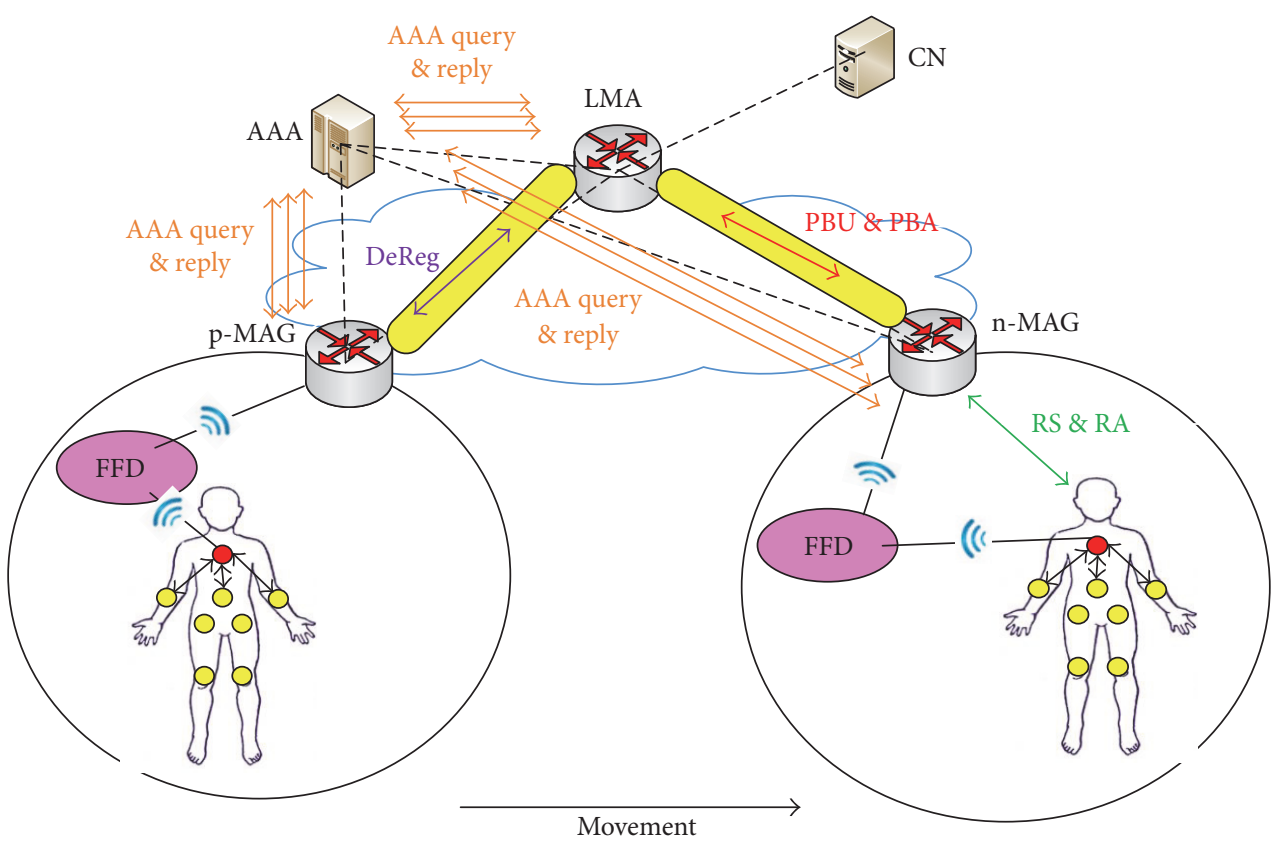

FFD Full-Function Device

- Coordinator

○ Sensors

FIgURE 3: Existing PMIP-Coordinator mobility schemes. 


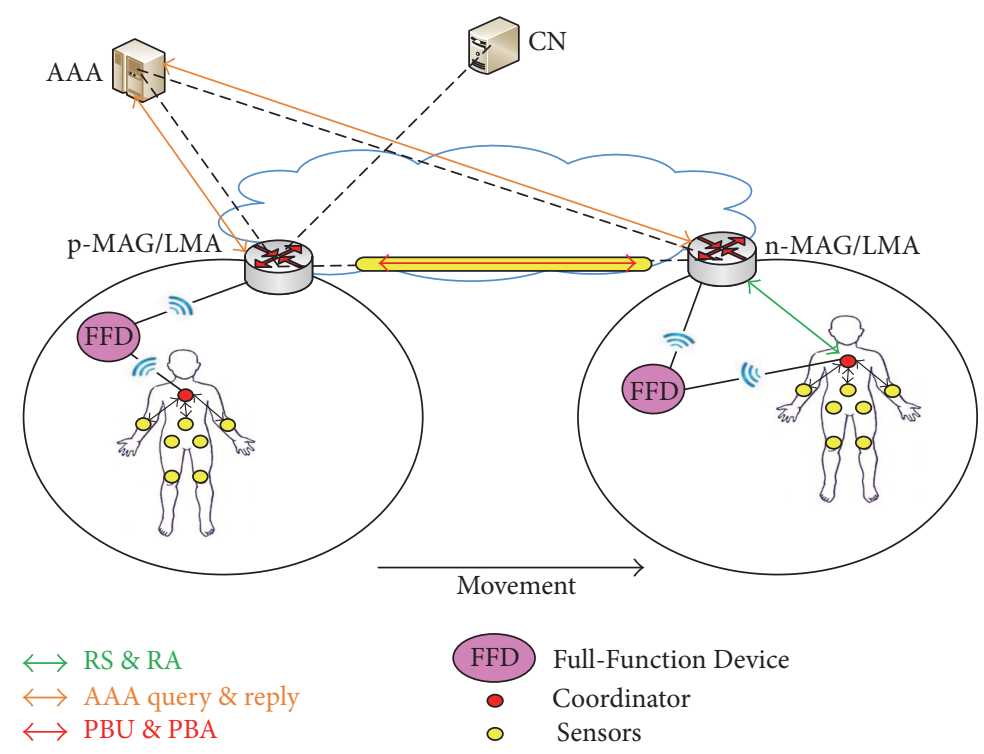

FIGURE 4: Network model for the proposed scheme.

\section{Distributed Group-Based Mobility Management Scheme}

This section first introduces the network model of the proposed scheme and then describes the initial registration and handover operations.

3.1. Network Model. Figure 4 shows the network model for the proposed scheme. We consider a group of IPv6 over Lowpower Wireless Personal Area Network (6LoWPAN) sensors attached to the human body. One of them acts as a coordinator, and only the coordinator can exchange the control signaling messages with the Mobile Access Gateway (MAG) on behalf of the other sensors. The 6LoWPAN domain contains a Full-Function Device (FFD). In the proposed scheme, the Local Mobility Anchor (LMA) function is implemented by each MAG, and the handover operation is performed between two neighboring MAGs without the help of LMA for the group of sensors. Each MAG maintains the information of the group of sensors and aggregates the AuthenticationAuthorization-Accounting ( $A A A)$ query messages to the AAA server in the authentication process. Each MAG does not perform the binding update and deregistration operation with LMA, since it has the LMA functionality.

Initially, the coordinator communicates with the correspondent node $(\mathrm{CN})$ in the previous Mobile Access Gateway (p-MAG) domain, and then it moves to a new Mobile Access Gateway (n-MAG) by handover.

3.2. Initial Registration. The aim of the registration phase is to reduce the amount of control messages. Figure 5 illustrates the initial registration of the proposed scheme. When a group of body sensors enter a PMIP domain and the coordinator is attached to MAG/LMA, it sends an aggregated Router Solicitation (RS) message, containing the information on group, MN-IDs, and Link-Layer Addresses (LLAs), to MAG/LMA (Step 1).

Upon reception of this $R S$ message from the coordinator, MAG/LMA sends an aggregated $A A A$ query message with all LLAs for authentication to the AAA server. The aggregated $A A A$ query message is different from the previous schemes, in that it contains LLAs of a group of sensors.

After authentication, the AAA server responds with a "single" AAA reply message to MAG/LMA (Steps 2 and 3). After that, MAG initiates the DHCP solicitation procedure to request home network prefixes (HNPs) for the body sensors one by one. Then, the DHCP server replies with the unique HNPs for each body sensor. The MAG creates the binding cache entry; store the home network prefix information. The DHCP server also configures the respective Home-ofAddress (HoA) from those prefixes and sends it to MAG. Then, the MAG/LMA responds to the coordinator with an RA message as a response to the $R S$ message (Step 4).

3.3. Handover Operations. The body sensors perform active scans periodically with nearby FFDs by sending a beacon request. The nearby FFDs receive the beacon request from the body sensors and advertise the beacon message containing their MAG-IDs to the body sensors. The body sensors receive the beacon message and decide whether it still resides in the same MAG or has moved to another MAG by comparing the current MAG-IDs with the previous MAG-IDs. If the current MAG-ID and previous MAG-ID are the same, then the movement represents intra-PAN mobility. On the other hand, if the current MAG-ID is different from the previous MAGID, then the body sensors are able to detect its movement from the old MAG to the new MAG.

Figure 6 shows the detailed signaling flow for the handover procedure. We now assume that the coordinator changes its point of attachment in the same network domain. When the coordinator is detached from p-MAG/LMA and 


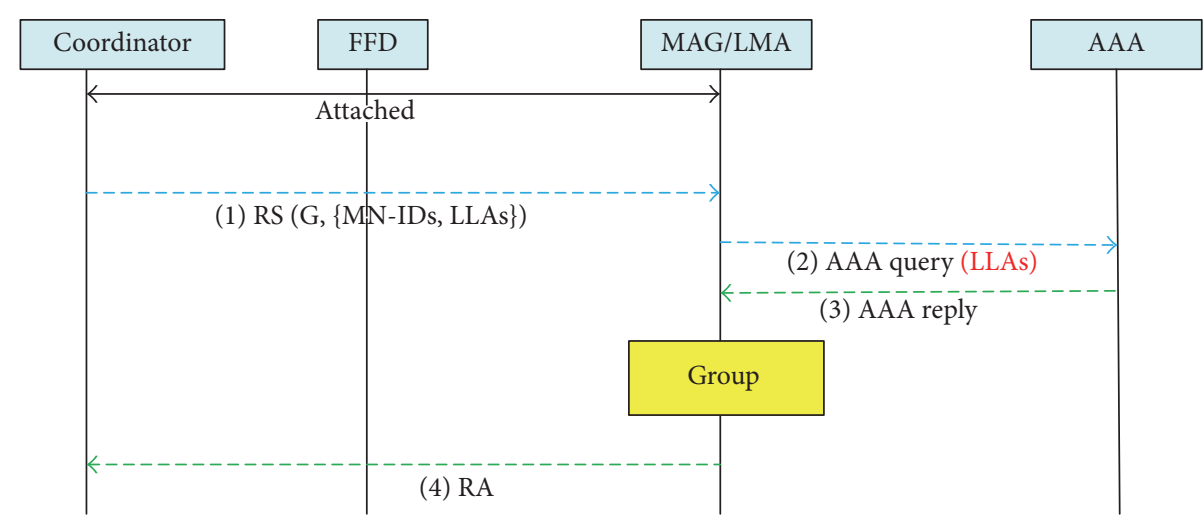

FIGURE 5: Initial registration.

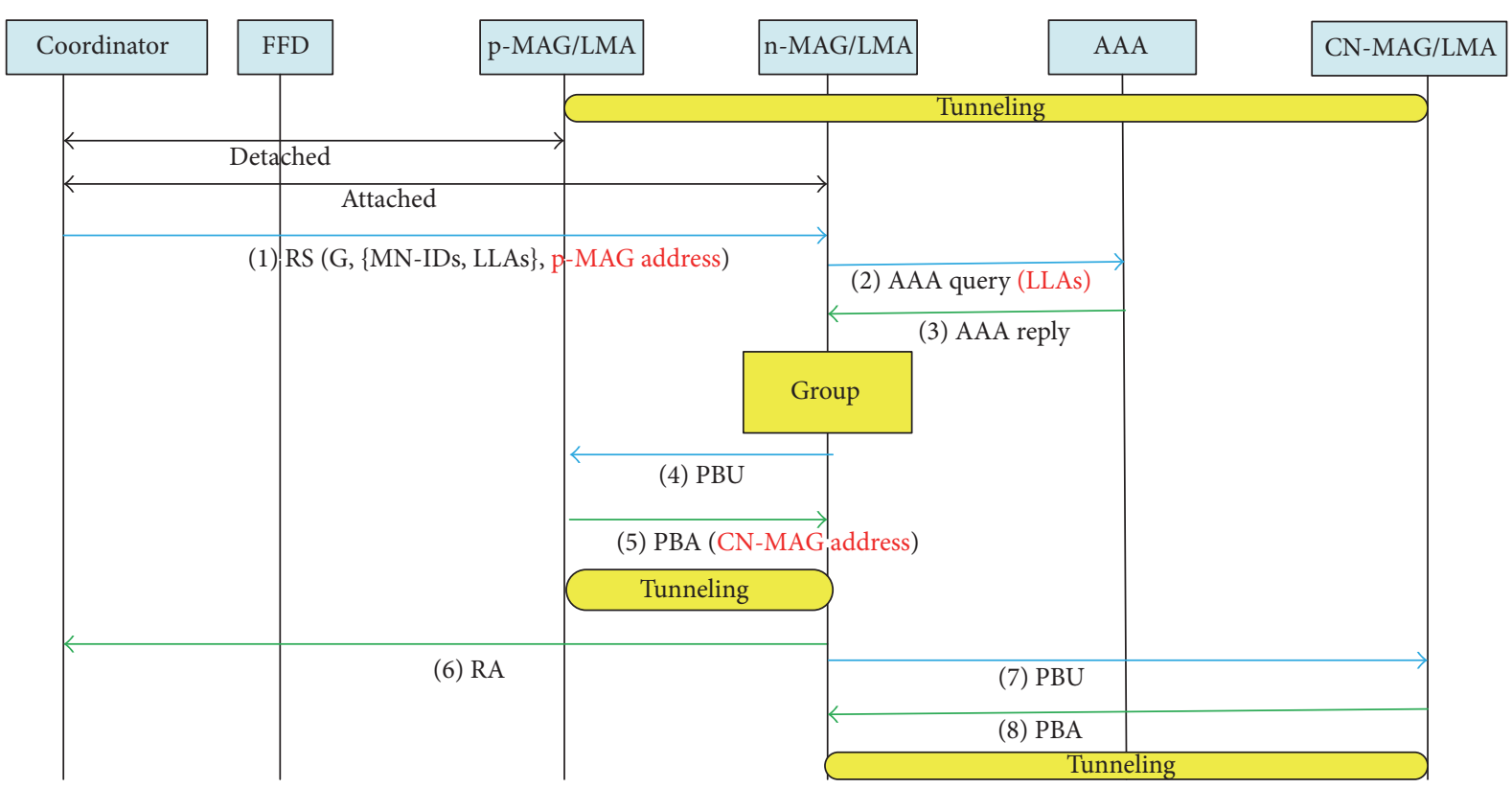

FIGURE 6: Handover operations.

attached to n-MAG/LMA, then the coordinator sends an aggregated $R S$ message to n-MAG/LMA (Step 1). After that, n-MAG/LMA will exchange the aggregated $A A A$ query and reply messages with AAA server (Steps 2 and 3). After authentication, n-MAG sends a Proxy Binding Update (PBU) message to p-MAG/LMA for handover control (Step 4). Now, n-MAG/LMA obtains the location of p-MAG/LMA from the $R S$ message. After that, the p-MAG/LMA responds with Proxy Binding Ack (PBA) to n-MAG/LMA (Step 5). The $P B A$ message shall include the information of CN-MAG/ LMA address, which is recorded into the mapping table of p-MAG/LMA. So, the handover tunnel is established between p-MAG/LMA and n-MAG/LMA for data forwarding. After establishment of handover tunnel, n-MAG responds to the coordinator with a Router Advertisement (RA) message (Step 6).

Now, n-MAG/LMA sends a $P B U$ message to CN-MAG/ LMA for route optimization (Step 7). On reception of the
$P B U$ message, CN-MAG/LMA will update its mapping table and send a PBA to n-MAG/LMA (Step 8). n-MAG/LMA and $\mathrm{CN}-\mathrm{MAG} / \mathrm{LMA}$ will now use the optimized route.

\section{Performance Analysis}

For performance analysis, we compare the registration and handover delays for the four candidate mobility schemes: PMIP, PMIP-Group, PMIP-Coordinator, and the proposed scheme.

4.1. Analysis Model. We consider a network illustrated in Figure 7, in which each wired/wireless link is represented by bandwidth, latency, and average queuing delay. We adopt a generic model for Multiple Access Control (MAC) scheme to focus on the analysis of registration delay and handover delay associated with the proposed mobility scheme. 


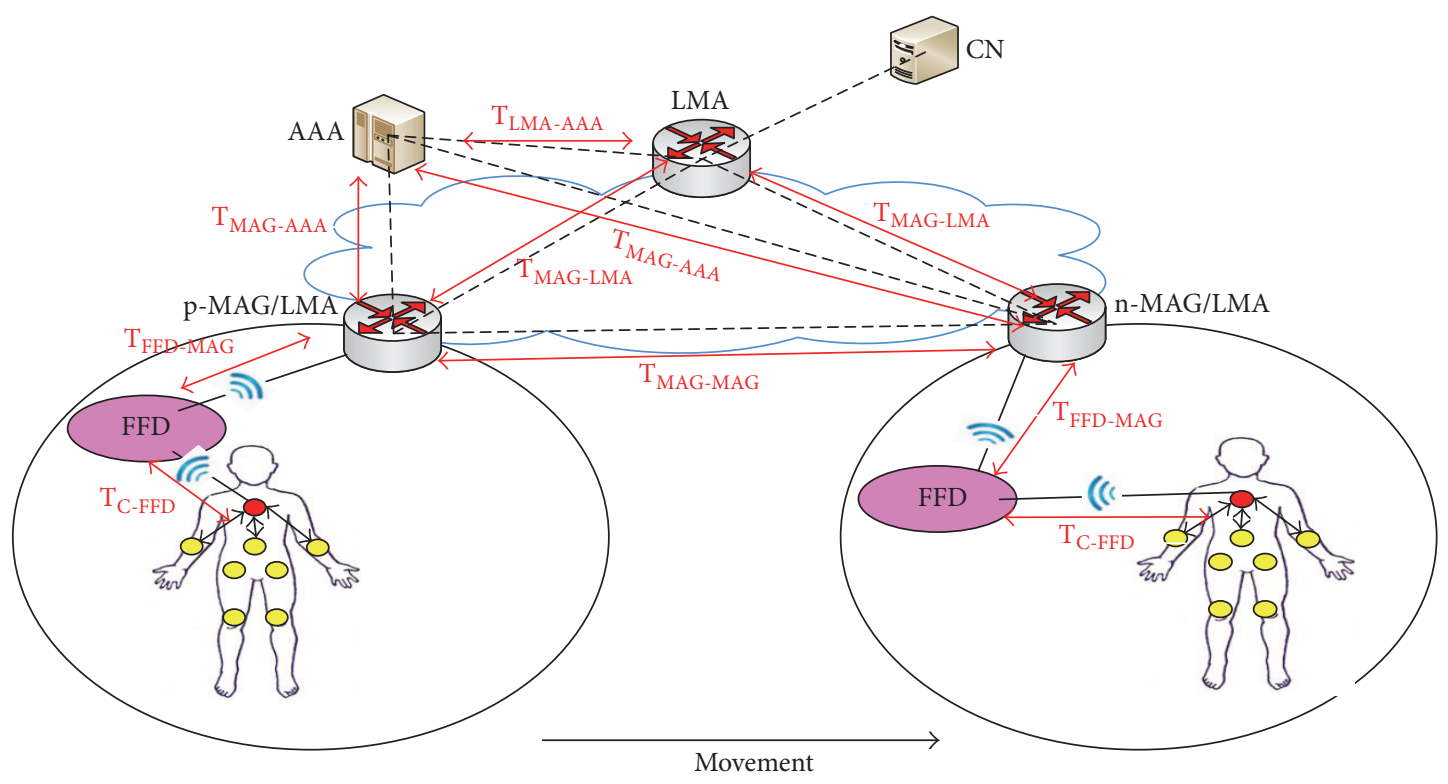

FIGURE 7: Network model for performance analysis.

We summarize the notations used in our analysis in the Parameters Used for Analysis.

In Figure 7 , we denote by $T_{x-y}(S)$ the transmission delay of a message with size $S$ sent from $x$ to $y$ via the "wireless" link, where each message can experience the failure at the probability of $q$ by using "iid" error model. Then, $T_{x-y}(S)$ can be expressed as $T_{x-y}(S)=[1 /(1-q)] \times\left[\left(S / B_{\mathrm{wl}}\right)+L_{\mathrm{wl}}\right]$. In the meantime, we denote by $T_{x-y}\left(S, H_{x-y}\right)$ the transmission delay of a message with size $S$ sent from $x$ to $y$ via the "wired" link, where $H_{x-y}$ represents the number of wired hops between node $x$ and node $y$. Then, $T_{x-y}\left(S, H_{x-y}\right)$ is expressed as $T_{x-y}\left(S, H_{x-y}\right)=H_{x-y} \times\left[\left(S / B_{w}\right)+L_{w}+T_{q}\right]$.

4.2. Registration Delay (RD). As shown in Figure 1, when the body sensors are attached to a MAG, they send $R S$ messages to $M A G$ via the coordinator and FFD. After that, MAG performs the AAA operations for authentication for all body sensors. Then, MAG performs the $P B U$ operation with LMA for all body sensors. Now, LMA performs the AAA operations with AAA server for all body sensors. After authentication, LMA responds with the $P B A$ message to MAG. Now, MAG will respond with the $R A$ messages to all of the body sensors. Accordingly, we get the RD of PMIP as follows:

$$
\begin{aligned}
& \mathrm{RD}_{\text {PMIP }}=N_{S} \times\left\{2 T_{\mathrm{C}-\mathrm{FFD}}\left(S_{c}\right)+2 T_{\mathrm{FFD}-\mathrm{MAG}}\left(S_{c}\right)\right. \\
& +2 T_{\text {MAG-AAA }}\left(S_{c}\right)+2 T_{\text {MAG-LMA }}\left(S_{c}\right) \\
& \left.+2 T_{\text {LMA-AAA }}\left(S_{c}\right)\right\} .
\end{aligned}
$$

As shown in Figure 2, the whole operations of PMIPGroup are the same as those of PMIP. Different from PMIP, the PMIP-Group scheme uses the aggregated $P B U$ and $P B A$ messages between LMA and MAGs. Accordingly, we get the RD of PMIP-Group as follows:

$$
\begin{aligned}
& \mathrm{RD}_{\text {PMIP-Group }}=N_{S} \times\left\{2 T_{\text {C-FFD }}\left(S_{c}\right)+2 T_{\text {FFD-MAG }}\left(S_{c}\right)\right. \\
& \left.+2 T_{\text {MAG-AAA }}\left(S_{c}\right)+2 T_{\text {LMA-AAA }}\left(S_{c}\right)\right\} \\
& +2 T_{\text {MAG-LMA }}\left(S_{c}\right) .
\end{aligned}
$$

As shown in Figure 3, the PMIP-Coordinator uses the aggregated $R S$ and $R A$ messages between MAG and the coordinator. Thus, we get the RD of PMIP-Coordinator as

$$
\begin{aligned}
& \mathrm{RD}_{\text {PMIP-Coordinator }} \\
& =2 T_{\mathrm{C}-\mathrm{FFD}}\left(S_{c}\right)+2 T_{\mathrm{FFD}-\mathrm{MAG}}\left(S_{c}\right)+2 T_{\text {MAG-LMA }}\left(S_{c}\right) \\
& \quad+N_{S} \times\left\{2 T_{\text {MAG-AAA }}\left(S_{c}\right)+2 T_{\text {LMA-AAA }}\left(S_{c}\right)\right\} .
\end{aligned}
$$

In the proposed scheme, when the coordinator is attached to $\mathrm{n}-\mathrm{MAG}$, then it sends an aggregated $R S$ message on behalf of all body sensors to n-MAG by way of FFD. After that, nMAG performs the AAA operations with AAA server. Based on this, n-MAG performs the authentication process and responds with an aggregated $R A$ message to the coordinator. Thus, we get the RD of the proposed scheme as follows:

$$
\begin{aligned}
\mathrm{RD}_{\text {Proposed Scheme }}= & 2 T_{\mathrm{C}-\mathrm{FFD}}\left(S_{c}\right)+2 T_{\mathrm{FFD}-\mathrm{MAG}}\left(S_{c}\right) \\
& +2 T_{\text {MAG-AAA }}\left(S_{c}\right) .
\end{aligned}
$$

4.3. Handover Delay (HD). The handover delay is defined as the gap between the time that body sensors cannot receive the packets from $\mathrm{p}-\mathrm{MAG}$ and the time that body sensors receive the first packet from n-MAG. 
As shown in Figure 1, when the body sensors are detached from p-MAG, then p-MAG will send DeReg messages to LMA for body sensors. When the body sensors are attached to n-MAG, they send $R S$ messages to n-MAG by way of the coordinator and FFD. After that, n-MAG performs the AAA query and reply operations with AAA server for authentication for all body sensors, and then n-MAG performs the $P B U$ operations with LMA for all body sensors. Now, LMA performs the AAA query/reply operations with AAA server for all body sensors. After authentication, LMA responds with the $P B A$ message to n-MAG. The handover tunnel will be established between n-MAG and LMA. After the tunnel establishment, n-MAG responds with the $R A$ messages to all body sensors. Accordingly, we get the HD of PMIP as follows:

$$
\begin{aligned}
& \mathrm{HD}_{\text {PMIP }}=N_{S} \times\left\{2 T_{\mathrm{C}-\mathrm{FFD}}\left(S_{c}\right)+2 T_{\text {FFD-MAG }}\left(S_{c}\right)\right. \\
& +2 T_{\text {MAG-AAA }}\left(S_{c}\right)+4 T_{\text {MAG-LMA }}\left(S_{c}\right) \\
& \left.+2 T_{\text {LMA-AAA }}\left(S_{c}\right)\right\}+T_{\text {MAG-LMA }}\left(S_{d}\right) .
\end{aligned}
$$

As shown in Figure 2, the PMIP-Group scheme uses the aggregated DeReg and $P B U / P B A$ messages. Accordingly, we get the HD of PMIP-Group as follows:

$$
\begin{aligned}
& \mathrm{HD}_{\text {PMIP-Group }}=N_{S} \times\left\{2 T_{\text {C-FFD }}\left(S_{c}\right)+2 T_{\text {FFD-MAG }}\left(S_{c}\right)\right. \\
& \left.+2 T_{\text {MAG-AAA }}\left(S_{c}\right)+2 T_{\text {LMA-AAA }}\left(S_{c}\right)\right\} \\
& +4 T_{\text {MAG-LMA }}\left(S_{c}\right)+T_{\text {MAG-LMA }}\left(S_{d}\right) .
\end{aligned}
$$

As shown in Figure 3, the PMIP-Coordinator scheme uses the aggregated $R S$ and $R A$ messages between MAG and the coordinator. Accordingly, we can get the HD of PMIPCoordinator as follows:

$$
\begin{aligned}
& \mathrm{HD}_{\text {PMIP-Coordinator }} \\
& =2 T_{\text {C-FFD }}\left(S_{c}\right)+2 T_{\text {FFD-MAG }}\left(S_{c}\right)+4 T_{\text {MAG-LMA }}\left(S_{c}\right) \\
& +N_{S} \times\left\{2 T_{\text {MAG-AAA }}\left(S_{c}\right)+2 T_{\text {LMA-AAA }}\left(S_{c}\right)\right\} \\
& \quad+T_{\text {MAG-LMA }}\left(S_{d}\right) .
\end{aligned}
$$

In the proposed scheme, when the coordinator is attached to n-MAG, it sends an aggregated $R S$ message on behalf of body sensors to n-MAG by way of FFD. After that, nMAG performs the AAA operations with the AAA server by aggregation. Then, n-MAG exchanges the $P B U$ and $P B A$ messages with p-MAG for establishment of a handover tunnel. After tunnel establishment, n-MAG responds with an aggregated $R A$ message to the coordinator. Accordingly, we get the HD of the proposed scheme as follows:

$$
\begin{aligned}
\text { HD }_{\text {Proposed Scheme }}= & 2 T_{\text {C-FFD }}\left(S_{c}\right)+2 T_{\text {FFD-MAG }}\left(S_{c}\right) \\
& +2 T_{\text {MAG-AAA }}\left(S_{c}\right) \\
& +2 T_{\text {MAG-MAG }}\left(S_{c}\right) \\
& +T_{\text {MAG-MAG }}\left(S_{d}\right) .
\end{aligned}
$$

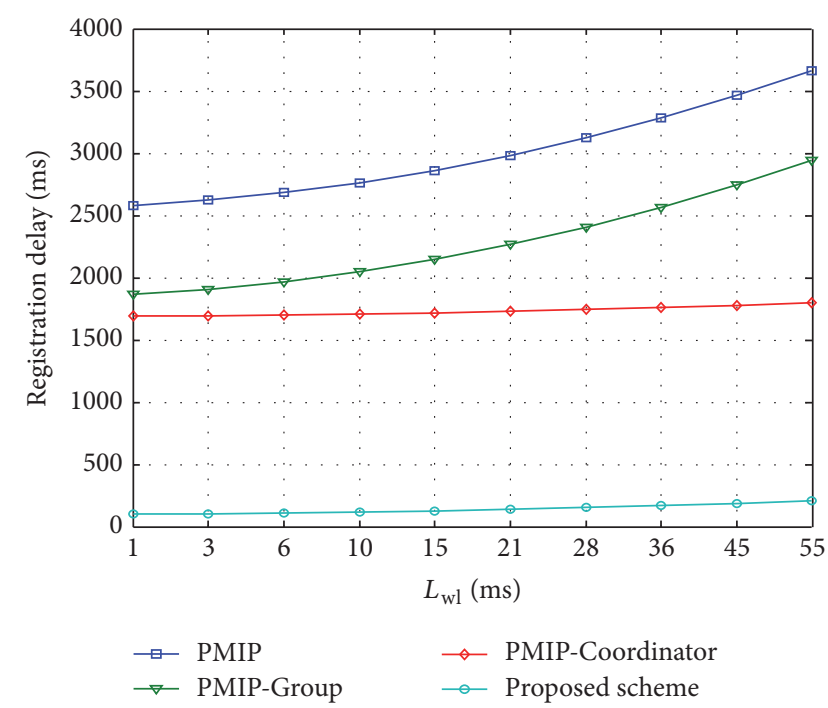

FIGURE 8: Impact of $L_{\mathrm{wl}}$ on registration delay.

\section{Numerical Results and Discussion}

Based on the analysis given so far, we now compare the performances of the candidate schemes.

For numerical analysis, by referring to [24], the default value of each parameter is configured as follows: $H_{\text {MAG-LMA }}=$ $H_{\text {MAG-MAG }}=5, H_{\text {MAG-AAA }}=H_{\text {LMA-AAA }}=5, L_{\mathrm{wl}}=10 \mathrm{~ms}$, $L_{w}=2 \mathrm{~ms}, q=0.5, T_{q}=5 \mathrm{~ms}, N_{S}=10, S_{c}=96$ bytes, $S_{d}=$ 200 bytes, $B_{\mathrm{wl}}=11 \mathrm{Mbps}$, and $B_{w}=100 \mathrm{Mbps}$. Among these parameters, we note that $L_{\mathrm{wl}}, T_{q}$, and $N_{s}$ may depend on the network conditions. Thus, we will compare the performances of candidate schemes by varying those parameter values.

5.1. Registration Delay. Figure 8 shows the impact of wireless link delay $\left(L_{\mathrm{wl}}\right)$ on registration delay. From the figure, we can see that the registration delay linearly increases as $L_{\mathrm{wl}}$ gets larger, for all candidate schemes. It is shown that the proposed scheme gives better performance than the existing schemes. This is because the proposed scheme uses the LMA functionality over MAG, and thus the PBU and PBA operations are not required between LMA and MAG. In the proposed scheme, the MAG also performs aggregated AAA query and reply operations.

Figure 9 compares the registration delay for different average queuing delay $\left(T_{q}\right)$ at each node. It is shown in the figure that the registration delay linearly increases as $T_{q}$ gets larger, for all candidate schemes. We can see that the proposed scheme gives the best performance among the candidate schemes. This is because each MAG implements the LMA functionality.

Figure 10 illustrates the impact of the number of sensors $\left(N_{s}\right)$ on registration delay. In the figure, the handover delay linearly increases as $N_{s}$ gets larger, for the existing schemes. It is shown that the proposed scheme is not affected by $N_{s}$. This is because the amounts of the AAA query and reply messages for each registration are constant no matter what the number of the body sensors is. Besides, the proposed scheme also uses 


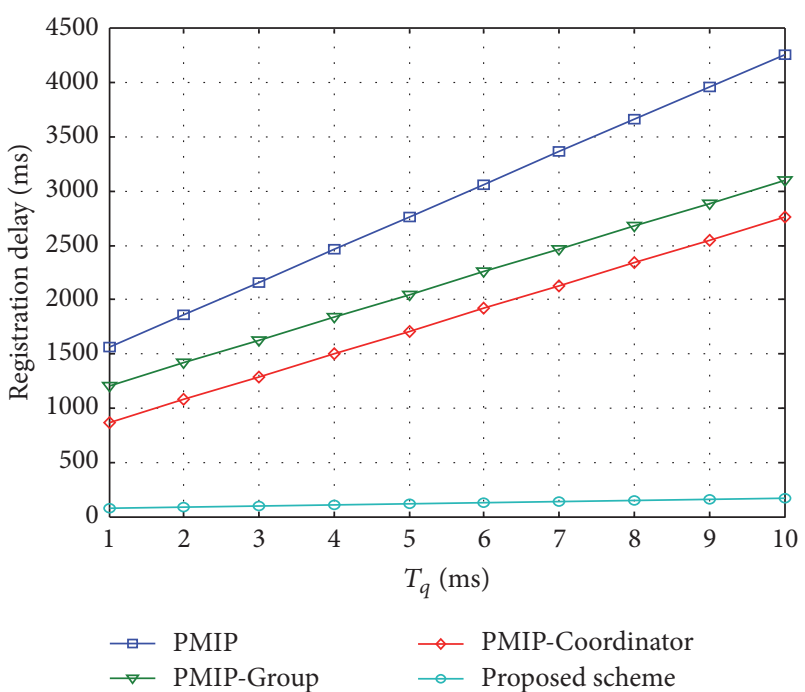

Figure 9: Impact of $T_{q}$ on registration delay.

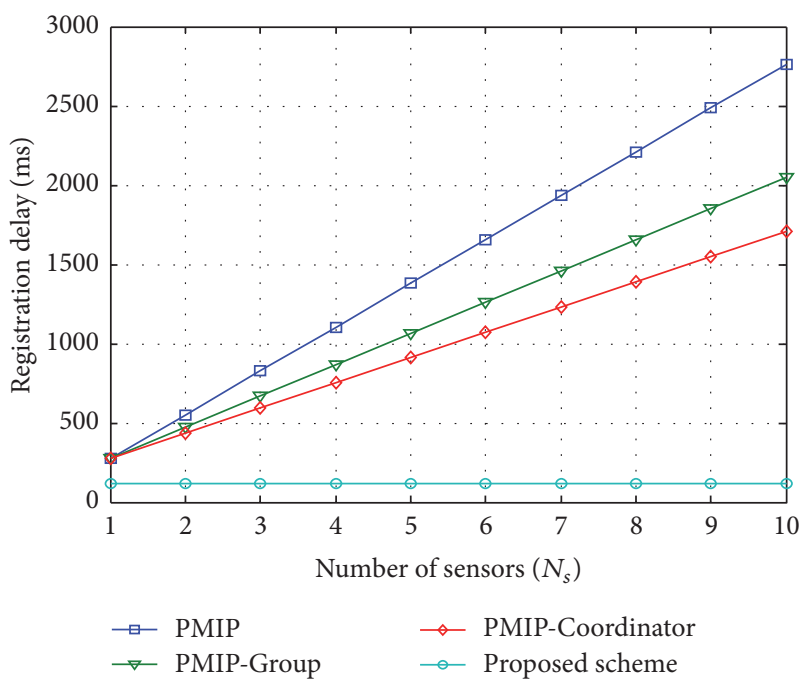

FIgURE 10: Impact of $N_{s}$ on registration delay.

the LMA functionality over MAG, and thus the PBU and PBA operations are not required between LMA and MAG. Hence, each body sensor needs to transmit a smaller amount of control messages and incurs lower registration delay as the number of body sensors increases.

5.2. Handover Delay. Figure 11 illustrates the impact of wireless link delay $\left(L_{\mathrm{wl}}\right)$ on handover delay. In the figure, the handover delay linearly increases as $L_{\mathrm{wl}}$ gets larger, for all candidate schemes. It is shown that the proposed scheme gives better performance than the existing schemes. This is because the LMA function is implemented by each MAG and the handover operation is performed between two neighboring MAGs without the help of LMA.

Figure 12 shows the impact of average queuing delay $\left(T_{q}\right)$ on handover delay. It is shown in the figure that the handover

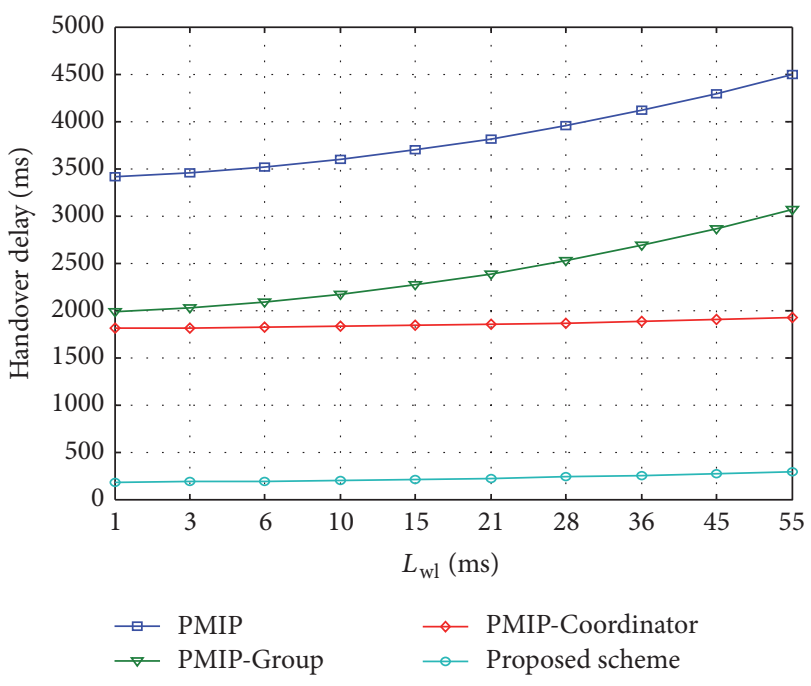

FIgURE 11: Impact of $L_{\mathrm{wl}}$ on handover delay.

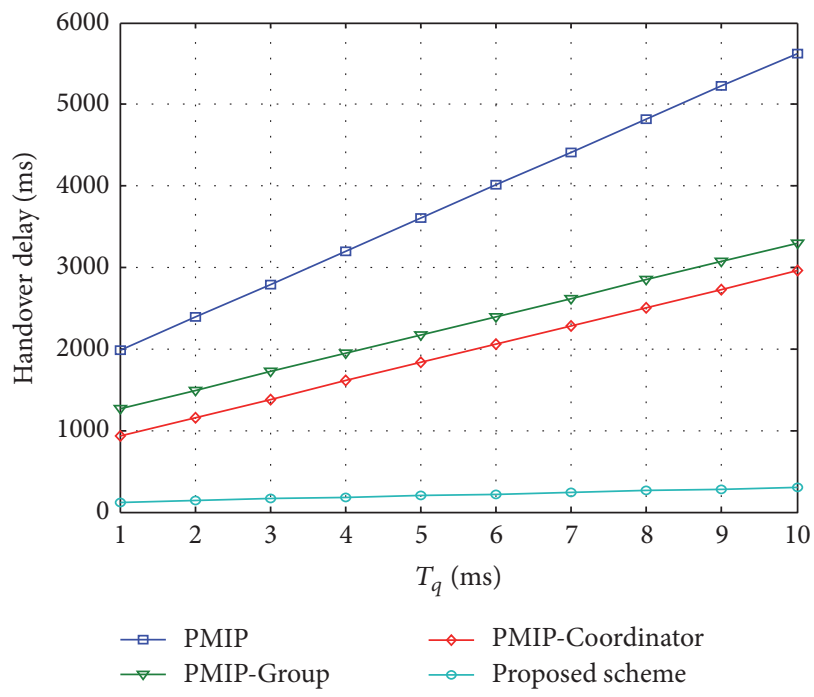

FIGURE 12: Impact of $T_{q}$ on handover delay.

delay linearly increases as $T_{q}$ gets larger, for all the candidate schemes. We can see that the proposed scheme gives the best performance among the candidate schemes.

Figure 13 shows the impact of the number of sensors $\left(N_{s}\right)$ on handover delay. In the figure, the handover delay linearly increases as $N_{s}$ gets larger, for the existing schemes. It is shown that the proposed scheme is not affected by $N_{s}$. This is because the amounts of the AAA query and reply messages for each handoff are constant no matter what the number of the body sensors is. Besides, the proposed scheme also implemented the LMA functionality over MAG, and the handover operation is performed between two neighboring MAGs without the help of LMA and thus the deregistration operations are not required between LMA and p-MAG. Hence, each body sensor needs to transmit a smaller amount of control messages and incurs lower handover delay as the number of body sensors increases. 


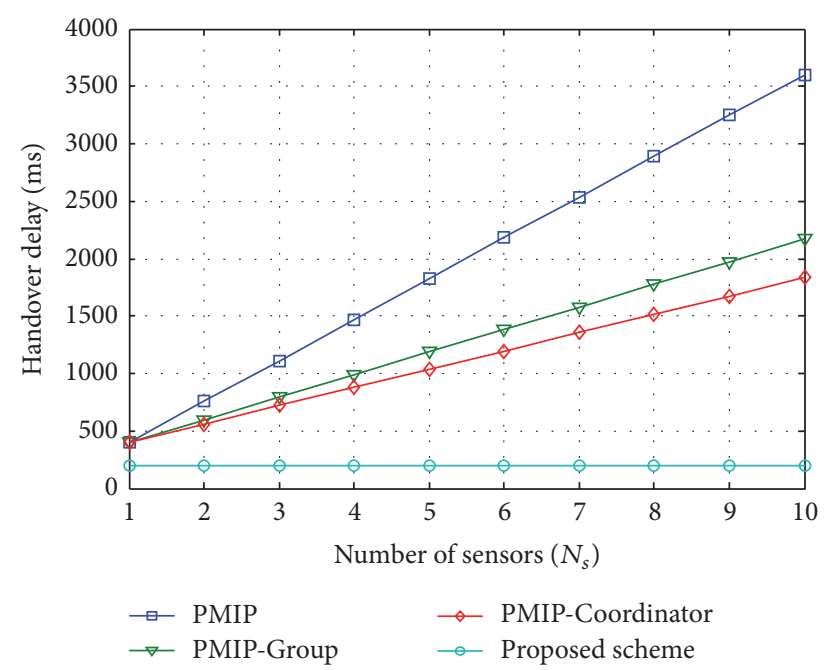

Figure 13: Impact of $N_{s}$ on handover delay.

\section{Conclusions}

In this paper, we proposed a distributed group-based mobility management scheme in 6LoWPAN-based wireless body area networks. In the proposed scheme, the LMA function is implemented by each MAG, and the handover operation is performed between the two neighboring MAGs. Furthermore, the MAG aggregates the AAA query messages for the group of moving sensors. From the numerical results, we see that the proposed scheme can provide better performance than the existing schemes in terms of registration and handover delays.

\section{Parameters Used for Analysis}

$S_{c}: \quad$ Size of control packets (bytes)

$S_{d}: \quad$ Size of data packets (bytes)

$N_{S}$ : Number of sensors in the domain

$B_{w}$ : Wired link bandwidth (Mbps)

$B_{\mathrm{wl}}$ : Wireless bandwidth (Mbps)

$L_{w}$ : Wired link delay (ms)

$L_{\mathrm{wl}}$ : Wireless link delay (ms)

$H_{a-b}$ : Hop count between nodes $a$ and $b$ in the network

$q$ : Wireless link failure probability

$T_{q}$ : Average queuing delay at each node.

\section{Conflicts of Interest}

The authors declare that they have no conflicts of interest.

\section{Acknowledgments}

This research was funded by the Deanship of Scientific Research, University of Dammam, Saudi Arabia (2016-410PYSS). This work was also supported by the 2016 Yeungnam University Research Grant.

\section{References}

[1] M. Ghamari, B. Janko, R. S. Sherratt, W. Harwin, R. Piechockic, and C. Soltanpur, "A survey on wireless body area networks for ehealthcare systems in residential environments," Sensors, vol. 16, no. 6, pp. 831-864, 2016.

[2] R. Cavallari, F. Martelli, R. Rosini, C. Buratti, and R. Verdone, "A survey on wireless body area networks: technologies and design challenges," IEEE Communications Surveys and Tutorials, vol. 16, no. 3, pp. 1635-1657, 2014.

[3] R. Sharma, H. S. Ryait, and A. K. Gupta, "Wireless body area nework-a review," Research Cell, vol. 17, 2016.

[4] J. Xing and Y. Zhu, "A survey on body area network," in Proceedings of the 5th International Conference on Wireless Communications, Networking and Mobile Computing (WiCOM '09), September 2009.

[5] M. Gohar, J.-G. Choi, and S.-J. Koh, "An ID/locator separation based group mobility management in wireless body area network," Journal of Sensors, vol. 2015, Article ID 537205, 12 pages, 2015.

[6] N. Kushalnagar, G. Montenegro, and C. Schumacher, "IPv6 over low-power wireless personal area networks (6LoWPANs): overview, assumptions, problem statement, and goals," RFC, 4919, IETF, 2007.

[7] D. Johnson, C. Perkins, and J. Arkko, "Mobility support in IPv6," RFC 3775, IETF, 2004.

[8] D. Saha, A. Mukherjee, I. S. Misra, and M. Chakraborty, "Mobility support in IP: a survey of related protocols," IEEE Network, vol. 18, no. 6, pp. 34-40, 2004.

[9] H. Soliman and C. Castelluccia, Hierarchical Mobile IPv6 Mobility Management, British Royal Flying Corp, 2005.

[10] V. Devarapalli, R. Wakikawa, A. Petrescu, and P. Thubert, "Network Mobility (NEMO) basic support protocol," RFC 3963, 2005.

[11] Y.-S. Chen, C.-S. Hsu, and H.-K. Lee, "An enhanced group mobility protocol for 6lowpan-based wireless body area networks," IEEE Sensors Journal, vol. 14, no. 3, pp. 797-807, 2014.

[12] S. Hyeon, Y.-H. Han, H.-B. Lee, and H.-Y. Choi, "Empirical performance evaluation of IETF mobile IPv6 and proxy mobile IPv6," Proceedings of the international conference on mobile technology,applications, and systems, mobility'08 Article 68, 2008.

[13] M. Chen, S. Gonzalez, A. Vasilakos, H. Cao, and V. C. M. Leung, "Body area networks: a survey," Mobile Networks and Applications, vol. 16, no. 2, pp. 171-193, 2011.

[14] R. S. H. Istepanian, E. Jovanov, and Y. T. Zhang, "Introduction to the special section on $\mathrm{m}$-Health: beyond seamless mobility and global wireless health-care connectivity," IEEE Transactions on Information Technology in Biomedicine, vol. 8, no. 4, pp. 405414, 2004.

[15] G. Montenegro, N. Kushalnagar, J. Hui, and D. Culler, "Transmission of IPv6 packets over IEEE 802.15.4 networks," RFC 4944, 2007.

[16] M. Shin, T. Camilo, J. Silva, and D. Kaspar, "Internet-draft6LoWPAN mobility," Internet-Draft, vol. 2, pp. 5-10, 2007.

[17] L. M. L. Oliveira, A. F. de Sousa, and J. J. P. C. Rodrigues, "Routing and mobility approaches in IPv6 over LoWPAN mesh networks," International Journal of Communication Systems, vol. 24, no. 11, pp. 1445-1466, 2011.

[18] M. M. Islam and E.-N. Huh, "Sensor proxy mobile IPv6 (SPMIPv6) - a novel scheme for mobility supported IP-WSNs," Sensors, vol. 11, no. 2, pp. 1865-1887, 2011. 
[19] J. Kim, R. Haw, E. J. Cho, C. S. Hong, and S. Lee, "A 6LoWPAN sensor node mobility scheme based on proxy mobile IPv6," IEEE Transactions on Mobile Computing, vol. 11, no. 12, pp. 2060-2072, 2012.

[20] G. Bag, M. T. Raza, K.-H. Kim, and S.-W. Yoo, "LoWMob: intraPAN mobility support schemes for 6LoWPAN," Sensors, vol. 9, no. 7, pp. 5844-5877, 2009.

[21] J. H. Kim, C. S. Hong, and T. Shon, "A lightweight NEMO protocol to support 6LoWPAN," ETRI Journal, vol. 30, no. 5, pp. 685-695, 2008.

[22] Y. Li, Y. Jiang, H. Su, D. Jin, L. Su, and L. Zeng, "A groupbased handoff scheme for correlated mobile nodes in proxy mobile IPv6," in Proceedings of IEEE Global Telecommunications Conference, GLOBECOM 2009, pp. 1-6, December 2009.

[23] R. Chai, Y.-L. Zhao, Q.-B. Chen, T. Dong, and W.-G. Zhou, "Group mobility in 6LoWPAN-based WSN," in Proceedings of International Conference on Wireless Communications and Signal Processing, WCSP 2010, pp. 1-5, October 2010.

[24] C. Makaya and S. Pierre, "An analytical framework for performance evaluation of IPv6-based mobility management protocols," IEEE Transactions on Wireless Communications, vol. 7, no. 3, pp. 972-983, 2008. 


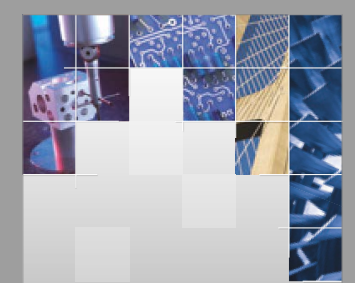

\section{Enfincering}
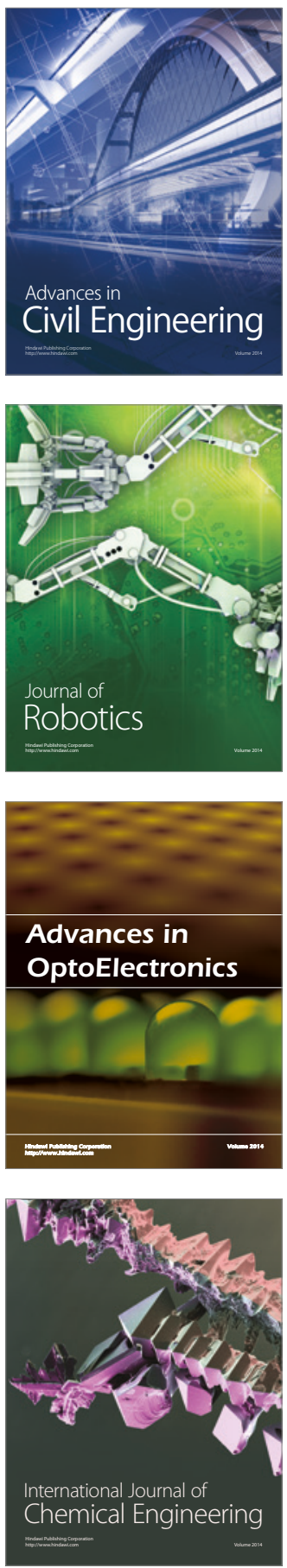

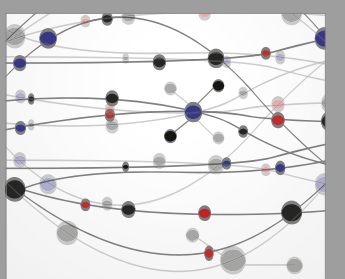

The Scientific World Journal

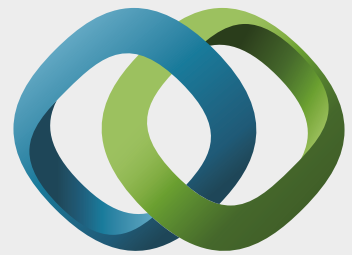

\section{Hindawi}

Submit your manuscripts at

https://www.hindawi.com
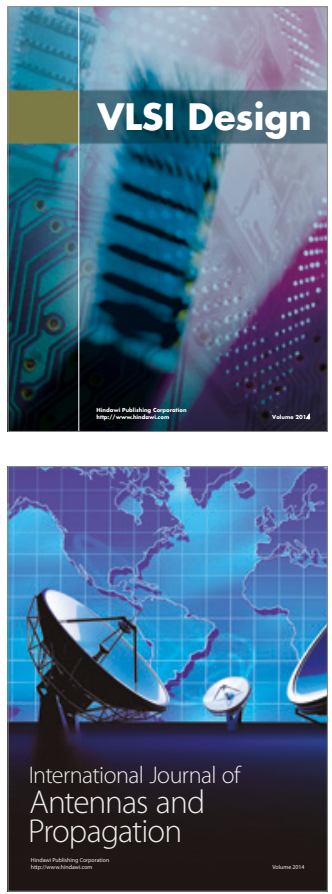

\section{Rotating}

Machinery
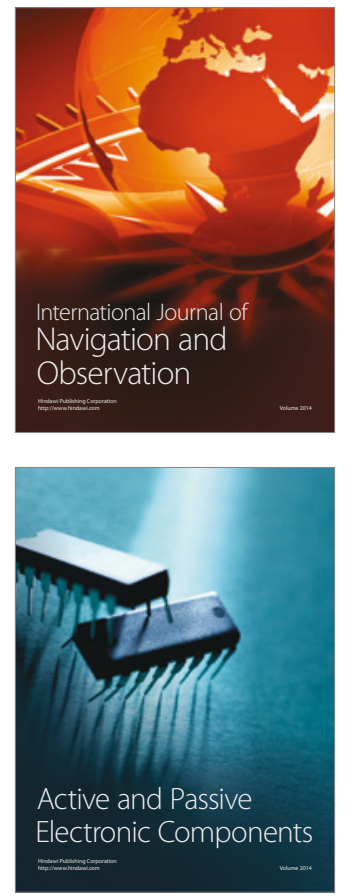
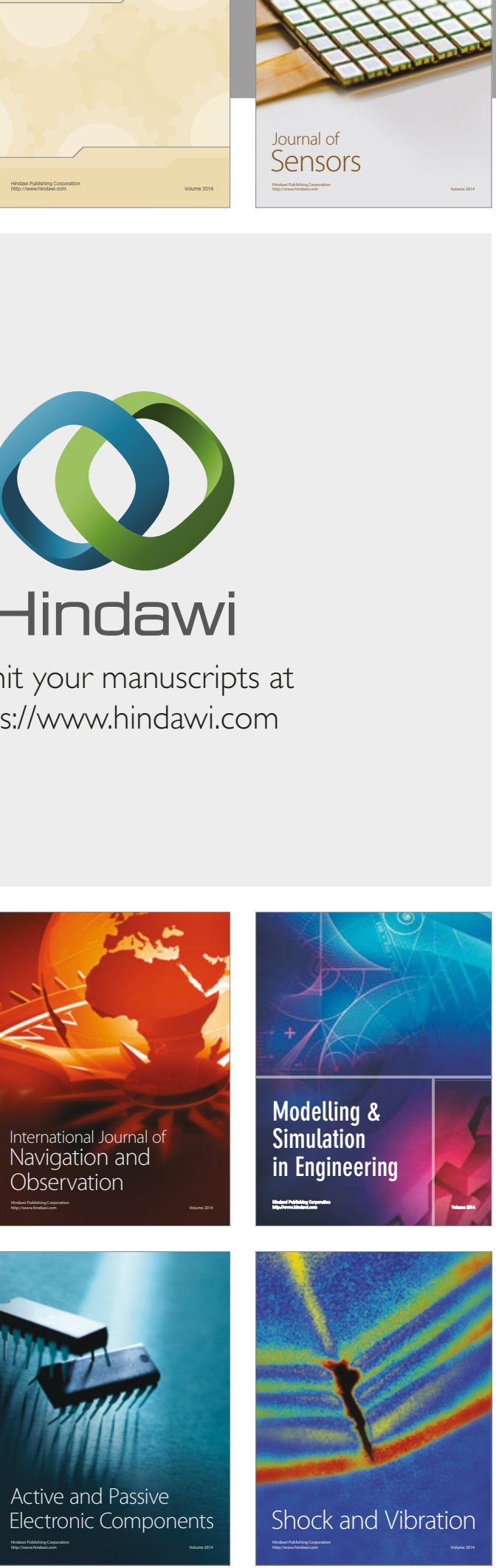
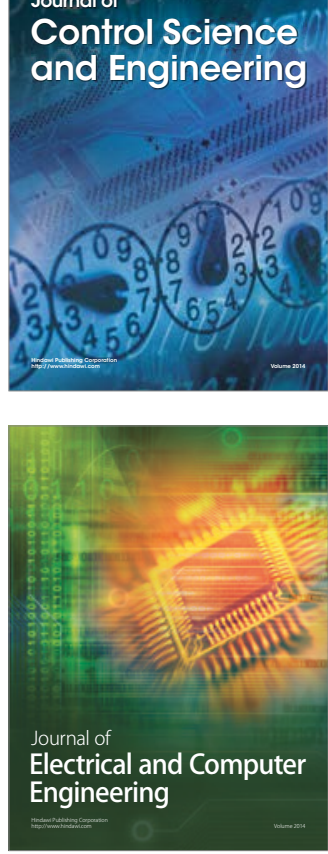

Distributed

Journal of

Control Science

and Engineering
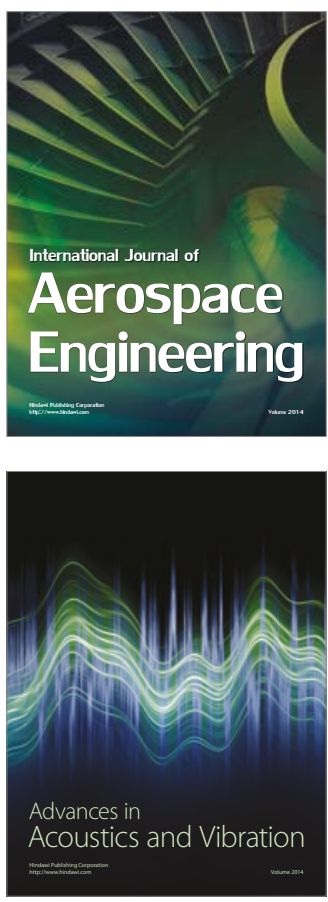

Sensor Networks 